wird: Im Tarnkleid der Verharmlosung der nationalsozialistischen Verbrechen. Die nachfolgende Sentenz aus dem Urteil, mit der der BGH seine Ausführungen zusammenfaßt, könnte über den konkreten Anlaß und den ausschließlichen Bezug auf "Volksgruppen " hinaus interpretiert werden als ein beispielgebendes Programm einer historischen Konkretisierung des Begriffs und Schutzbereichs der Menschenwürde, die ultimativ alle jene sozialen Gruppen, die auf Grund rassischer, religiöser, weltanschaulicher oder politischer Merkmale in der Geschichte zum Objekt staatlicher Ausgrenzung, Unterdrückung und Verfolgung gemacht wurden, vor jedwedem Ansatz erneuter Diskriminierung auf Grund eben derselben kollektiven Vorurteile in Schutz nimmt: „Wird der Einzelne durch die Entindividualisierung und Entwürdigung seiner Volksgruppe in der Vergangenheit so hervorgehoben, daß gerade deshalb seine Ehre die Achtung vor seiner Gruppe fordert, dann ist der personale Gehalt jenes Geschehens, um dessentwillen er diesen Achtungsanspruch hat, von seiner Persönlichkeit nicht zu trennen. Der Versuch, diese Vorgänge zu rechtfertigen, zu beschönigen oder abzustreiten, enthält eine Mißachtung auch seiner Person" (S. 9). Bezogen nicht nur auf die Juden, sondern auch auf Zigeuner, Zeugen Jehovas, ja auch Homosexuelle und selbst Kommunisten wäre dies als die Formulierung eines Verständnisses der Menschenwürde zu betrachten, welches sich die Ent-Stigmatisierung der Opfer staatlicher Gewaltverbrechen, die Tabuisierung der Reproduktion erneut entsprechender Feindbilder zur Aufgabe setzte. Freilich: Der Gedanke an staatlich verordnete Lernprozesse aus der Geschichte, an staatlich verordnete Geschichtsbilder schreckt; die Beschränkung der öffentlichen Kommunikation durch staatlich garantierte Schutzbereiche der Menschenwürde muß - sofern man sie überhaupt zulassen will - strikte Ausnahme bleiben. Jedenfalls aber sollten durch diese Ausführungen des BGH die staatlichen Institutionen daran erinnert werden, nicht ihrerseits lediglich selektiy aus der Geschichte zu lernen und partiell doch wieder in die Fußstapfen vergangener »Entindividualisierung und Entwürdigung« zu treten.

Thomas Blanke

\title{
Dokumentation zum Rationalisierungsschutz
}

\section{A. Zur Bedeutung der neueren Rationalisierungsschutzabkommen}

Während der Prosperitätsphase der Bundesrepublik entwickelten sich die Kernmaterien $^{1}$ des Arbeits- und Sozialrechts zu relativ ${ }^{2}$ selbständigen und voneinander abgesonderten Gebieten. Arbeitsschutz und Sozialpolitik wurden das Feld des Gesetzgebers und der sozialpolitischen Lobby. Arbeitsvertrags- und kampfrecht wurden das Feld der tarifschließenden Verbände. Die Betriebsverfassung wurde das Feld von jeweiligem Arbeitgeber und Betriebsrat. Augenscheinlich hat sich dies in der Phase der Stagnation und der Krise ab 1974 verändert. Krisen aktualisieren und verstetigen die in der Lohnarbeiterrolle angelegten Reproduktionsrisiken. Mit diesen Risiken aber sind die drei genannten Regelungsbereiche (und die mit ihnen

\footnotetext{
I Die Einteilung ist angelehnt an Karl Korsch, Arbeitsrecht für Betriebsräte, I922 (Frankfurt I968).

$2 \mathrm{Daß}$ es sich bei dieser Verselbständigung nur um eine relative handelt, worüber der organisatorische, justizielle und gesetzgeberische Zusammenhang nicht übersehen werden soll, sei nur am Rande vermerkt.
} 
beschäftigten Personen und Institutionen) gleichermaßen konfrontiert. Konkretisie-

ren läßr sich diese Beobachtung am Rationalisierungsproblem.

Der Ausgangspunkt von Rationalisierungsmaßnahmen ist der Betrieb. ${ }^{3}$ Dort werden arbeitsorganisatorische und/oder technische Veränderungen von Arbeitsvollzügen eingeleitet. Dort versuchen Beschäftigte und deren betriebliche Interessenvertreter, den negativen Folgen - wie Arbeitsplatzverlust, Lohneinbußen, Qualifikationsschwund, Arbeitsintensivierung usw. - entgegenzutreten, Entlassungen und Herabgruppierungen abzuwehren, auf Arbeitstempi und -bedingungen Einfluß zu nehmen, Sozialpläne oder betriebliche Requalifizierungs- und Umsetzungsprogramme durchzusetzen. Nur zu oft erweisen sich diese betrieblichen Gegenmaßnahmen als nicht durchsetzbar oder wirkungslos. Dann werden die Rationalisierungsmaßnahmen zu einem Problem der Gewerkschaften. Tarifpolitik kann dann nicht mehr auf die Verkaufsbedingungen der Arbeitskraftverkäufer beschränkt bleiben - wie in zwei Jahrzehnten vorherrschender ${ }^{4}$ gewerkschaftlicher Lohn- und Manteltarifpolitiks -, sondern muß den betrieblichen Entstehungsort des Rationalisierungsproblems einbeziehen. Die Rationalisierungsfolgen zeigen sich schließlich auch außerhalb der Produktionssphäre - als Arbeitslosigkeit, Frühinvalidität usw. - und strukturieren sich dauerhaft zu einem Kreis von Problemgruppen, -branchen, und -regionen. Damit ist der Staat mit dem Rationalisierungsproblem konfrontiert. Sozialpolitik, die lediglich allgemeine Mindestreproduktionsbedingungen setzt, reicht dann nicht mehr aus. Qualifikations- und Requalifikationsprogramme, problemorientierte Schutz- und Hilfsprogramme, die gleichfalls auf die betriebliche Ebene zielen, werden notwendig.

Zum gesellschaftlichen Problem sind Rationalisierungsfolgen in der Bundesrepublik erst in der zweiten Hälfte der 6oer Jahre und verstärkt ab 1974 geworden. Auch vorher schon waren Beschäftigte und Betriebsräte damit konfrontiert. Aber die rationalisierungsbedingten "Freisetzungen « wurden innerbetrieblich oder sektoral kompensiert, solange die Beschäftigung im industriellen, im tertiären Sektor sowie im öffentlichen Dienst ausgeweitet wurde. Nach der Krise I $966 / 67$ begann zunächst im industriellen Produktionsbereich, dann phasenverschoben seit Beginn der joer Jahre im Angestellten-, Dienstleistungsbereich und dem öffentlichen Dienst der systematische Einsatz von Automaten und elektronischer Datenverarbeitung. Die dadurch bedingten »Freisetzungen « wurden nun nicht mehr durch anderweitige Ausweitung der Beschäftigung kompensiert. Dies erst löste gewerkschaftliche Diskussionen $^{6}$ und erste Gegenmaßnahmen ${ }^{7}$ aus. Auch die Reformvorhaben der ersten

3 Die Verwaltungen des öffentlichen Dienstes werden hier nicht gesondert erwähnt, doch treffen die meisten hier angestellten Uberlegungen auch auf sie zu.

4 Eine Ausnahme war bereits relativ frühzeitig der Lohnrahmentarifvertrag II für die gewerkschaftlichen Arbeitnehmer der Metallindustrie Nordwürttemberg/Nordbaden gültig ab 1. 11. 1973. Dieser sah Erholungszeiten und Mindesttaktzeiten vor. Zu betonen ist jedoch, daß dieser Tarifvertrag - entgegen anderslautenden Erwartungen - sich nicht über das Tarifgebiet hinaus ausgebreitet hat; ferner wurde er im Tarifgebiet selber auch nicht vollständig in die betriebliche Wirklichkeit umgesetzt: vgl. Der Lohnrahmentarifvertrag II in der betrieblichen Praxis, SOFI/Göttingen, November 9977.

s Vgl. E. Schmidt, Ordnungsfaktor oder Gegenmacht. Die politische Rolle der Gewerkschaften, Frankfurt/Main 1971; Bergmann/Jacobi/Müller-Jentsch, Gewerkschaften in der BRD. Gewerkschaftliche Lohnpolitik zwischen Mitgliederinteressen und ökonomischen Sachzwängen, Frankfurt und Köln 1975 .

6 Vgl. z. B. Automation und technischer Fortschritt in Deutschland und den USA. Ausgewählte Beiträge zu einer internationalen Arbeitstagung der Industriegewerkschaft Metall für die Bundesrepublik Deutschland (3.-5. Juli 1963), Frankfurt/Main 1963; Automation. Risiko und Chance, Beiträge zur 2. Internationalen Arbeitstagung der Industriegewerkschaft Metall für die Bundesrepublik Deutschland über Rationalisierung, Automatisierung und technischen Fortschritt, 16.-19. März 196s in Oberhausen, Frankfurt ig6s.

7 Z. B. Abkommen zum Schutz der Arbeitnehmer (Arbeiter und Angestellte) vor Folgen der Rationalisierung für die Metallindustrie vom 27. 5./25.6. 1968 . 
Phase der sozialliberalen Koalition sind von der Rationalisierungserfahrung und dem Versuch ihrer Bewältigung geprägt. Das Arbeitsförderungs- und das Berufsbildungsgesetz von 1969 sowie die Novelle zur Betriebsverfassung 1972 streben an, Arbeitslosigkeit zu verhindern und Qualifizierungs- und Requalifizierungsprozesse zu begünstigen. Sowie also das Rationalisierungsproblem zum gesellschaftlichen Problem wurde, hat es die genannten Kernmaterien des Arbeits- und Sozialrechts ihrer relativen Selbständigkeit enthoben und gewissermaßen am Problem "gebündelt «.

In der Phase nach Beginn der Wirtschaftkrise 1974 ist jedoch eine paradoxe Wende eingetreten, die in der Frage der Rationalisierungsbewältigung zunehmend offene Flanken spürbar werden läßt. Seither breiten sich Mikroprozessoren im industriellen, Rechnersteuerung und Automaten im industriellen wie auch im Angestelltenund Verwaltungsbereich aus. Deren negative Beschäftigungseffekte verbinden sich noch mit denjenigen der konjunkturellen Entwicklung. Wird also einerseits das Rationalisierungsproblem brisanter und drängender, so erweisen sich zunehmend die betrieblichen und staatlichen Versuche der Folgenabwehr als wirkungslos. Daß sie auf betrieblicher Ebene scheitern, liegt auch an den begrenzten Einwirkungsmöglichkeiten und Durchsetzungsmitteln, über die die Beschäftigten und Betriebsräte verfügen. ${ }^{8}$ Meistens aber scheitern sie daran, daß Beschäftigte wie Betriebsräte - sich einzelkapitalistischer Rationalität beugend - die Konkurrenzfähigkeit »ihres« jeweiligen Unternehmens zu sichern suchen und deshalb betriebliche Rationalisierungsprozesse ungeachtet gesellschaftlicher Auswirkungen eher unterstützen als bekämpfen. Auch die Wirtschafts- und Sozialpolitik der zweiten Phase der sozialliberalen Koalition läßt keine Bewältigung der Rationalisierungsfolgen erwarten. Diese zweite Phase begann mit den Investitionszulagen 1974, die Rationalisierungsprozesse ohne Auflagen begünstigten, und dem Haushaltsstrukturgesetz 1975, das den Sozialetat kürzte. Sie zielt auf die Rekonsolidierung der Einzelkapitale, auf die Steigerung der internationalen Konkurrenzfähigkeit der westdeutschen Wirtschaft, auf Anti-Inflations-Politik, auf die Konsolidierung der Staatsfinanzen und allenfalls punktuell auf Hilfsprogramme ab. Sie schränkt unternehmerische Autonomie nicht ein, unterstützt auch nicht direkt die Beschäftigteninteressen. ${ }^{9}$ So wird die Rationalisierungsbewegung staatlicherseits keineswegs behindert, sondern durch eine breite Palette von Mitteln - Investitionsprämien, Steuervergünstigungen, Forschungs- und Entwicklungsprogramme, Konzentrationsförderung, jeweils ohne beschäftigungspolitische Auflagen - gestützt und vorangetrieben.

Die Gewerkschaften stehen also in der Rationalisierungsfrage vor zwei offenen Flanken. Auf sich gestellt, werden weder die betriebliche noch die staatliche Rationalisierungspolitik den Beschäftigteninteressen gerecht. Daß allein eine eigene gewerkschaftliche Rationalisierungspolitik Abhilfe schaffen, darüber hinaus zum Motor betrieblicher und staatlicher Aktivitäten werden kann, wird im gewerkschaftlichen Bereich zunehmend erkannt. In den letzten Jahren ist das Vertrauen in staatliche Sozialpolitik sichtlich geschwunden, die Wirksamkeit institutionalisierter

8 Hierzu die Úbersicht in meinem Artikel: Moderne Maschinenstürmerei? Zur Gegenwehr bei Rationalisierungen, in: Duhm/Mückenberger, Arbeitskampf im Krisenalltag, Berlin 1977, S. 83 ff.

9 Das Mitbestimmungsgesetz 1976 fällt etwas aus dem Rahmen dieser Einschätzung heraus, weitet es doch in gewissem Umfang die Eingriffe in unternehmerische Autonomie aus. Betrachtet man jedoch das Gesetz im Spiege] des Urteils des Bundesverfassungsgerichts vom I. März 1977, so geht daraus hervor, daß die institutionalisierte Unternehmensbestimmung weniger den unmittelbaren Beschäftigteninteressen als vielmehr der Verbesserung der „Funktionsfähigkeit “ der Unternehmen unter veränderten sozialen Bedingungen dient. Insoweit kann das Gesetz durchaus als Bestandteil der wirtschaftspolitischen Strategie der Rekonsolidierung der Einzelkapitale angesehen werden. 
Unternehmensmitbestimmung wird skeptischer betrachtet, ${ }^{10}$ die zu bekämpfenden Rationalisierungsfolgen werden genauer erfaßt, die bisherigen Rationalisierungsschutzabkommen realistischer beurteilt." Tarifpolitische Konzepte und Durchsetzungschancen werden stärker auf das Rationalisierungsproblem bezogen. ${ }^{12}$ Gewiß begegnen diese Ansätze des Umdenkens großen innergewerkschaftlichen Widerständen. In den Gewerkschaftsgremien dominiert - nicht anders als bei Betriebsrä ten und Politikern der Regierungskoalition - die Perspektive der Problementlastung durch verbesserte Konkurrenzfähigkeit der westdeutschen Wirtschaft. Notwendig bleibt damit auch ihr Verhalten zum Rationalisierungsproblem widersprüchlich und inkonsequent, wo Rationalisierungsschutzbestrebungen die Konkurrenzfähigkeit der Unternehmen zu beeinträchtigen drohen. Doch keiner Gewerkschaft gelingt bruchlos, die "Mitgliederinteressen « in "Systemzwänge $" z u$ integrieren. ${ }^{13}$ Die Gewerkschaften beruhen auf freiwilliger Mitgliedschaft und Beitragszahlung und sind daher auf ein Mindestmaß an Loyalität der Mitgliedschaft und (um diese zu erzielen und zu erhalten) an aktuell wirksamer Interessenpolitik angewiesen. Daraus entsteht ein Spannungsverhältnis, das nicht nur die zunehmenden Aktivitäten von Gewerkschaften in Sachen Rationalisierung erklärt, sondern auch die unterschiedliche Rationalisierungspolitik verschiedener Gewerkschaften, gewerkschaftlicher Berufsgruppen oder Bezirke.

Auf Seiten der Rationalisierungsbetroffenen, ihrer Gewerkschaften und der die Rationalisierungsprozesse betreibenden Unternehmen lassen sich Bedingungen spezifizieren, die eine wirksame gewerkschaftliche Rationalisierungspolitik begünstigen bzw. erschweren: ${ }^{14}$

I. Auf Seiten der Betroffenen spielen Wille und Bereitschaft zur Durchsetzung von Rationalisierungsschutzabkommen eine Rolle. Diese werden beeinflußt durch a) das qualitative und quantitative Ausmaß sowie die inner- und überbetriebliche Vergleichbarkeit von Rationalisierungsprozessen und ihrer negativen Folgen;

b) die Art und Erheblichkeit der Folgen: Arbeitsplatzverlust und/oder Beeinträchtigung bei fortbestehendem Arbeitsverhältnis (Intensivierung, Lohn-/Qualifikationseinbuße);

Io Beispielsweise spielte die Forderung nach mehr Unternehmensmitbestimmung bei den auf Rationalisierung bezogenen Anträgen auf dem II. Ordentlichen Bundeskongreß des Deutschen Gewerkschaftsbundes (1978) kaum eine Rolle; vgl. die Anträge 183,185 und 188 , die angenommen wurden, und Anträge 187,184 und 186 , die als Material angenommen wurden: dokumentiert in Das Mitbestimmungsgespräch 1979 , Heft $5 / 6$, S. 1 ss ff

1 I Vgl. z. B. H. Pornschlegel, Tarifpolitische Perspektiven des technologischen Wandels, in: Technologietagung 1977. Strukturelle Arbeitslosigkeit durch technischen Wandel? Referate, gehalten auf der Technologietagung der IG Metall. 24./25. Mai 1977 Frankfurt/Main. Schriftenreihe der IG Metall Nr. 72, S. $103 \mathrm{ff}$. (I14); noch deutlicher ein vorläufiger Bericht der Abteilung Tarifpolitik beim IG Metall-Vorstand vom Oktober r 979: „Die herkömmlichen Rationalisierungsschutzabkommen sichern nicht den sozialen Status, sie mildern oder beseitigen für eine bestimmte Zeit negative Folgen. Sie verhindern nicht die Aushöhlung der Arbeitsinhalte und den Abbau von Qualifikationen. Sie verhindern somit auch nicht die negativen Folgen für die Arbeitsbedingungen, die Entgeltansprüche und den sozialen Status der Arbeitnehmer.\& (S. 60).

I2 So heißt es beispielsweise im Entwurf des neuen Grundsatzprogramms des Deutschen Gewerkschaftsbundes, beschlossen vom Bundesvorstand am 2. Oktober 1979: "Die technische Entwicklung führt nicht zwangsläufig zu humanen Arbeits- und Lebensbedingungen. Rationalisierung und Automation dürfen nicht die Beschäftigung, die Qualifikation, die Gesundheit und das Einkommen der Arbeitnehmer gefährden. Produktivitätsfortschritte, die allein durch eine Intensivierung der Arbeit angestrebt werden, sind zu bekämpfen. Technische und organisatorische Neuerungen dürfen erst dann durchgeführt werden, wenn die Interessen der Arbeitnehmer berücksichtigt und unzumutbare soziale Folgen ausgeschlossen sind. « Nach einer Reihe weiterer Forderungen zur »Humanisierung der Arbeit « heißt es dann: „Die Verwirklichung dieser Ziele ist eine wichtige Aufgabe der Tarifpolitik der Gewerkschaften. Diese muß durch eine entsprechende Betriebspolitik ergänzt werden.« (Hervorhebung U. M.)

13 Vgl. Bergmann u. a. (s. Fn. s), die dieses Spannungsverhältnis bereits im Titel ihrer Studie umreißen.

14 Es werden hier nur einige beachtenswerte Gesichtspunkte aufgeführt; deren Systematisierung ist in diesem Rahmen weder möglich noch beabsichtigt. 
c) die Fähigkeit und Bereitschaft der Betroffenengruppe (Facharbeiter, An- und Ungelernte, Frauen, Ausländer, Alte, Jugendliche, Angestellte, Beamte) zu kollektiver Aktion;

d) den gewerkschaftlichen Organisationsgrad und die Kampftraditionen der Betroffenen;

e) den Einfluß und die Repräsentanz der Betroffenengruppe in den gewerkschaftlichen Führungsgremien;

f) die aktuelle Arbeitsmarktsituation: ob eine industrielle Reservearmee vorhanden ist oder ob anderweitige akzeptable Arbeitsplätze existieren.

2. Auf Seiten der betroffenen Gewerkschaft spielen für die Frage, ob sie die Rationalisierungsbetroffenen aktiviert und unterstützt oder nicht, von den unter I.) aufgeführten Gesichtspunkten vor allem c) bis e) eine Rolle. Daneben wirken sich aus

a) die gewerkschaftlichen Durchsetzungsbedingungen angesichts der wirtschaftlichen Lage der Branche und der Gesamtwirtschaft;

b) die Art und der Erfolg der bisherigen - partnerschaftlichen oder konfliktorischen ${ }^{\text {is }}$ - Gewerkschaftsarbeit.

3. Auf Seiten der Unternehmen hängt die Bereitschaft zum bzw. der Widerstand gegen den Abschluß wirksamer Rationalisierungsschutzregelungen von den unter $\mathrm{I}$. f) und 2. a) und b) genannten Gesichtspunkten ab. Der Gesichtspunkt 2. a) läßt sich noch konkretisieren auf

a) die ökonomische Lage der betroffenen Branche und, damit zusammenhängend, den Grad der Erforderlichkeit der Rationalisierung;

b) den branchenspezifischen Lohnkostenanteil an den Gesamtkosten;

c) den bereits erreichten Grad an Arbeitsproduktivität und -intensität ${ }^{16}$ in den verschiedenen Unternehmensbereichen;

d) die zu erwartende Kostenbelastung bzw. die zu erwartende Erschwerung weiterer Rationalisierungsmaßnahmen durch das Abkommen.

An der spezifischen Bündelung solcher Einflußfaktoren lassen sich Verlauf und Ergebnis einiger Konflikte um Rationalisierungsschutzabkommen der letzten Zeit erklären. So wurden 1967 im hessischen Kautschukbereich, 1973 und 1978 im nordwürttembergisch-badischen Metallbereich, ebenfalls 1978 bundesweit im Druckbereich relativ neuartige Rationalisierungsbestimmungen ${ }^{17}$ durchgesetzt. In allen genannten Fällen waren dazu Streiks notwendig. Charakteristisch für diese Rationalisierungsschutzkonflikte waren in allen Fällen: massive Rationalisierungsbewegungen, hohe gewerkschaftliche Organisierung der Betroffenen und starke Repräsentanz in den Führungsgremien sowie konfliktorische Gewerkschaftspolitik einerseits, ${ }^{18}$ Krisenbedrohung der betroffenen Branchen und hoher Lohnkostenanteil andererseits. Dagegen sind etwa in der chemischen, der Mineralöl- und der Zigarettenindustrie gewissermaßen im Wege präventiver partnerschaftlicher Sozialpolitik einverständlich Regelungen eingeführt worden, die diejenigen in Problem-

is So die Begrifflichkeit von Bergmann u. a. (s. Fn. 5).

16 Zur Unterscheidung zwischen Steigerung der Arbeitsproduktivität und Arbeitsintensität vgl. Schmiede/Schudlich, Die Entwicklung der Leistungsentlohnung in Deutschland. Eine historisch-theoretische Untersuchung zum Verhältnis von Lohn und Leistung unter kapitalistischen Produktionsbedingungen, Frankfurt 1976; ferner J. H. Mendner, Technologische Entwicklung und Arbeitsprozeß. Zur reellen Subsumption der Arbeit unter das Kapital, Frankfurt 1975.

17 Als Rationalisierungsschutzbestimmungen werden hier nicht nur Bestimmungen aus Rationalisierungsschutztarifverträgen bezeichnet. Verschiedentlich haben auch Bestimmungen, die in anderen Tarifverträgen enthalten sind, Rationalisierungsschutzeffekt, wie z. B. beim Abgruppierungstarifvertrag von Nordwürttemberg/Nordbaden 1978 . Vgl. dazu die Beispiele in der folgenden Dokumentation.

18 Siehe oben Fn. 15 . 
branchen weit in den Schatten stellen. ${ }^{19}$ Dabei spielt gewiß eine Rolle, daß diese Branchen kaum verringertes Wachstum, fallenden bis geringfügigen Lohnkostenanteil, geringe gewerkschaftliche Aktivität, eher sozialpartnerschaftliche Betriebsratsund Gewerkschaftsarbeit aufweisen. Die genannten Einflußfaktoren bieten Anhaltspunkte zur Interpretation, sollen diese jedoch nicht ersetzen. Viele von ihnen sind nämlich mehrdeutig, wie sich an der Arbeitsmarktlage veranschaulichen läßt. Die günstige Arbeitsmarktlage der 6oer Jahre erklärt, warum die traditionellen Rationalisierungsschutzabkommen - etwa das bundesweite Metallabkommen von 1968 oder das Druckabkommen von I 967 - sich angesichts einer Großzahl offener Stellen mit finanzieller Kompensation des Arbeitsplatzverlustes begnügen. Ob aber eine ungünstige Arbeitsmarktlage, die Existenz einer industriellen Reservearmee die Bestrebungen nach Rationalisierungsschutz eher begünstigt (wie letzthin in der Druckindustrie) oder erschwert (wie in den lautlos niedergegangenen Leder- und Bekleidungsindustrien), läßt sich aus der Arbeitsmarktlage allein nicht erklären.

Bei aller Uneinheitlichkeit ist zweierlei den neueren gewerkschaftlichen Rationalisierungsschutzbestrebungen gemeinsam:

I. Die bisherige Lohn- und Manteltarifpolitik regelte wesentlich Lohnhöhe und -bemessung, Arbeitszeit und Urlaubsdauer, also Verkaufsbedingungen der Ware Arbeitskraft. Die neuen Regelungen greifen wenigstens vereinzelt in die Produktionsbeziehungen ein. Regelungen etwa über Mindesttaktzeiten, obligatorische Erholungspausen, über Arbeitsinhalte und Belastungsgrenzen, quantitative und qualitative Besetzungsregelungen ${ }^{20}$ machen nicht mehr gewissermaßen vor dem Fabriktor halt, sondern regeln den betrieblichen Gebrauch der Arbeitskraft.

2. Die traditionelle Rationalisierungsschutzpolitik kompensierte wesentlich finanziell den eingetretenen Arbeitsplatzverlust, ließ den Arbeitsplatz gleichsam "abkaufen«. Der Bestandsschutz war also auch marktförmig organisiert, auf Monetarisierung der Interessenbeeinträchtigung beschränkt. Davon unterscheiden sich die neueren Regelungen von Kündigungsverboten bei Verdienstsicherung für bestimmte Personengruppen, Statuierung echter Mitbestimmungsrechte bei Entlassungen, quantitative und qualitative Besetzungsregelungen. ${ }^{21}$ Sofern sie tariflich und betrieblich durchgesetzt werden, verhindern sie den Arbeitsplatzverlust, halten den geschützten Personenkreis ${ }^{22}$ in der Produktion.

Beide Regelungsbereiche begrenzen mit tariflichen Mitteln die Sach- oder Personalhoheit des betrieblichen Managements, was in dieser Form nicht üblich ist. Kein Wunder, daß diese Bestimmungen eine neue juristische Diskussion ${ }^{23}$ ausgelöst haben, ob dergleichen überhaupt tariflich regelbar ist oder nicht. Gemeinsam mit den traditionellen ist den neuen Regelungen jedoch, daß sie in der Regel ${ }^{24}$ den Rationalisierungsprozeß selbst als vom Unternehmen gesetztes Datum hinnehmen,

I9 Vgl. die Dokumentation.

20 Quantitative Besetzungsregelungen sind Tarifvertragsbestimmungen, die vorschreiben, wieviele Arbeiter an einem bestimmten Arbeitsplatz beschäftigt werden müssen. Qualitative Besetzungsregelungen sind Vorschriften, die bestimmen, welche Arbeitskräfte (mit welcher Qualifikation) an einem Arbeitsplatz beschäftigt werden dürfen.

2 I Siehe oben Fn. 20 und die Dokumentation; wAusdehnung von Mitspracherechten «.

22 Es sei nur am Rande darauf hingeweisen, daß sich tarifliche oder gesetzliche Schutzvorschriften für eine bestimmte Personengruppe durchaus auch zu deren Lasten auswirken können: vielfach werden die so "geschützten * Personengruppen entweder überhaupt nicht beschäftigt oder sie werden - ehe die Voraussetzungen des besonderen Schutzes vorliegen - entlassen. Systematisch kann auf diese Widersprüchlichkeit von Schutzbestimmungen hier nicht eingegangen werden.

23 Vgl. insbesondere D. Reuter, Zulässigkeit und Grenzen tarifvertraglicher Besetzungsregelungen, ZfA I 978, S. $1-44$.

24 Etwas anderes gilt nur für die bereits genannten Regelungen des Lohnrahmentarifvertrags II, soweit er direkte Vorschriften für den Arbeitsprozeß enthält. 
daß sie sich auch bei Eingriffen in die Produktion in der Regel auf die Folgenbewältigung beschränken.

Einige dieser Regelungen werden nachfolgend dokumentiert. Sie sind hier nur beschrieben und nach Entstehungs- und Inhaltsmerkmalen befragt worden. Gleichwohl drückt sich in ihnen eine neuartige Verschränkung der tariflichen und der betrieblichen Ebene aus, die der rechtstheoretischen Reflexion wert ist.

Ulrich Mückenberger

\section{B. Tarifvertragliche Regelungen zum Schutz der Beschäftigten bei Rationalisierungsmaßnahmen}

Angesichts der Fülle bereits bestehender Regelungen' kann die Dokumentation weder Vollständigkeit noch auch nur Repräsentativität für sich beanspruchen. Sie konzentriert sich - nach einer kurzen Betrachtung der tariflichen Definition von Rationalisierungsmaßnahmen (I.) - auf die Instrumente der Arbeitsplatzsicherung (II.) und der Einkommenssicherung (III.). Da Regelungen im Hinblick auf Qualifikationsveränderungen, Arbeitsbedingungen und -inhalte erst vereinzelt bestehen, ${ }^{2}$ sind sie in dieser Dokumentation nur am Rande berücksichtigt.

Die ersten gewerkschaftlichen Strategieansätze zum Rationalisierungsproblem am Ende der 6oer Jahre beschränkten sich darauf, einzelne Folgen zu betrachten und punktuell aufzugreifen. Entsprechend punktuell-reaktiv waren die tarifvertraglichen Regelungen zur Sicherung des sozialen Besitzstandes: Priorität von Umsetzungen und Umschulungen vor Entlassungen, Verlängerung der Kündigungsfristen und finanzielle Milderung der Kündigungsfolgen (dazu Dok. I, 5, 6, 8, 9, I I, I 2, I4, I7, 19). Ab Mitte der 7oer Jahre wurden die Rationalisierungsfolgen genauer aufgegriffen, wurden die einzelnen Schutztatbestände präzisiert und ausgebaut (dazu Dok, 7 , 10, $13,18,19,20)$. Ansatzweise wird heute auch der Versuch unternommen, auf den Arbeitsprozeß selbst und damit die Bedingungen der Rationalisierung gestaltend Einfluß zu nehmen (dazu Dok. 2, 3, 4), also die Folgen präventiv und nicht mehr nur reaktiv zu regeln.

Die dokumentierten Schutzregelungen sollen nicht über die Begrenztheit der Instrumente hinwegtäuschen. In der Bundesrepublik gelten derzeit lediglich für die Hälfte der beschäftigten Arbeiter und Angestellten Rationalisierungsschutzregelungen. ${ }^{3}$ Inwieweit diese betrieblich verwirklicht werden, ist schwer festzustellen.

\section{Begriffsbestimmung $»$ Rationalisierung*}

Die Rationalisierungsdefinitionen zeigen oft Defizite. Indem die Definition auf bestimmte Erscheinungsformen (z. B. merhebliche Umgestaltung der betrieblichen

I Vgl. hierzu z. B. Böhle, F./Lutz, B., Rationalisierungsschutzabkommen, Göttingen 1974, S. s ff.; Müller-Jentsch, W., Gewerkschaftliche Tarifpolitik gegen Rationalisierungsfolgen, in: Jacobi, O., u. a., Gewerkschaftspolitik in der Krise - Kritisches Gewerkschaftsjahrbuch $1977 / 1978$, Berlin I 978, S. 63 ff. Gerlach, G., Neuere tarifpolitische Strategien der Gewerkschaften in der BRD zur Sicherung von Arbeitsplätzen und Besitzständen, in: WSI - Mitteilungen 1979, S. 221 ff.

2 Z. B. Lohnrahmentarifvertrag II Nordbaden-Nordwürttemberg 1973, abgedruckt in: IG-Metal], Bezirksleitung Stuttgart (Hg.), Lohnrahmentarifvertrag II, MTV für die gewerblichen Arbeitnehmer, gültig ab I. 11.1973

3 Vgl. Sozialpolitische Informationen Nr. 14 des Bundesministeriums für Arbeit und Sozialordnung vom I2. 8. 1976. 
Organisation «) abstellt, werden bereits auf dieser Ebene bestimmte Rationalisierungsmaßnahmen ausgeklammert. So sind in den meisten Tarifverträgen die personal"wirtschaftlichen « Sparmaßnahmen, Prozesse der Intensivierung der Arbeit und Verschlechterung der Arbeitsbedingungen, Auslagerung und Streichung von Dienstleistungen (im öffentlichen Dienst) nicht erfaßt.

Die Beschränkung der Anwendbarkeit der Abkommen auf bestimmte Auswirkungen (z. B. personelle Maßnahmen der Entlassung, Umsetzung usw.) engt die Reichweite erheblich ein. Sie ist Ausdruck einer auf Arbeitsplatz- und Einkommenssicherung beschränkten Problemsicht. Arbeitsintensität und -bedingungen fallen auch hier wieder aus dem Schutzbereich heraus.

I. Abkommen zum Schutz der Arbeitnehmer (Arbeiter und Angestellte) vor Folgen der Rationalisierung für die Metallindustrie vom 16. 8. 1968:

$\$ 2$ Grundsatz

I. Die Rationalisierung unter Einschluß von Mechanisierung und Automatisierung als ein ständiger Prozeß der wirtschaftlichen und technischen Entwicklung schafft notwendige Voraussetzungen für das wirtschaftliche Wachstum und soll dazu beitragen, den erreichten Lebensstandard zu sichern und zu verbessern.

2. Durch die Rationalisierungsmaßnahmen können aber auch nachteilige Folgen für Arbeitnehmer entstehen. Die Tarifvertragsparteien streben deshalb mit diesem Abkommen an, solche nachteiligen Folgen, die durch Rationalisierungsmaßnahmen entstehen, nach Möglichkeit zu vermeiden oder zu mindern.

$\$ 3$ Anwendungsbereich

1. Rationalisierungsmaßnahmen im Sinne dieses Tarifvertrages, die Ansprüche der davon betroffenen Arbeitnehmer auslösen können, sind vom Arbeitgeber, auch aus Weisungsgebundenheit, veranlaßte

a) Änderung der Produktionsabläufe durch Einsatz von Anlagen und Maschinen mit größerer technischer Leistungsfähigkeit,

b) Änderungen der Arbeitstechniken durch Fertigungseinrichtungen mit größerer technischer Leistungsfähigkeit,

c) der Kostenersparnis dienende erhebliche Umgestaltungen der betrieblichen Organisation, soweit sie

aa) Umsetzungen, Versetzungen oder Umgruppierungen,

bb) Umschulungen oder

cc) Entlassungen

zur unmittelbaren Folge haben.

\section{Arbeitsplatzsicherung}

Arbeitsplatzsicherung ist einer der wesentlichsten Regelungskomplexe des tarifvertraglichen Rationalisierungsschutzes. Nachfolgend werden die unterschiedlichen Anknüpfungspunkte für eine Arbeitsplatzsicherung aufgeführt.

Mittelbarer Kündigungsschutz

Hier handelt es sich nicht um Regelungen, in denen ein Verbot bzw. eine Einschränkung von Kündigungsmöglichkeiten vereinbart wird, sondern um positive Bestimmungen über qualitative Arbeitsplatzbesetzung bzw. den Erhalt eines bestimmten Qualifikationsniveaus. Diese haben zwar regelmäßig einen bestimmten generellen Beschäftigungseffekt. Eine Kündigung des einzelnen Beschäftigten ist jedoch nicht ausgeschlossen.

a) In sog. *qualitativen Besetzungsregelungen* wird festgelegt, daß für einen bestimmten Zeitraum bestimmte Tätigkeiten nur von dafür ausgebildeten Fachkräften ausgeübt werden dürfen, auch wenn diese Tätigkeiten eine solche Qualifikation 
nicht mehr erfordern. Dieser Beschäftigungsgruppe wird bei Umstellungsmaßnahmen ein besonderer, zeitlich begrenzter Konkurrenzvorteil zugebilligt.

2. TV über die Einfübrung und Anwendung rechnergesteuerter Textsysteme in der Druckindustrie vom 20. 3. 1978.4

$\$ 2$ Arbeitsplatzsicherung

(1) Im rechnergesteuerten Textsystem werden Gestaltungs- und Korrekturarbeiten, das heißt

a) Gestaltung nichtstandardisierter Anzeigen,

b) Anzeigenseitenumbruch,

c) Anzeigenseitenschlußkorrektur,

d) Bildschirmkorrektur, jedoch mit Ausnahme der mit dem Redigieren verbundenen Korrekturvorgänge,

e) Textseitenumbruch

für einen Zeitraum von 8 Jahren nach Umstellung der jeweiligen Tätigkeit durch geeignete Fachkräfte der Druckindustrie, insbesondere Schriftsetzer, ausgeübt.

(2) Von der Verpflichtung nach Abs. 1 kann abgewichen werden, wenn

a) geeignete Fachkräfte der Druckindustrie am Arbeitsmarkt nicht verfügbar sind oder

b) dadurch die Arbeitsplätze unmittelbar betroffener Arbeitnehmer fortfallen würden.

$\$ 3$ Weiterbeschäftigung

Für die Texterfassung im rechnergesteuerten Textsystem sind vorrangig Fachkräfte der Druckindustrie (einschließlich der am Perforator Beschäftigten) des Unternehmens zu beschäftigen, deren Arbeitsplatz durch die Einführung des rechnergesteuerten Textsystems entfällt, sofern die entsprechende Tätigkeit vorher nicht von anderen Arbeitnehmern durchgeführt wurde. Den Fachkräften der Druckindustrie sind die Angestellten des Unternehmens gleichzustellen, deren Arbeitsplatz durch die Einführung des Systems entfällt.

b) Bei sog. "quantitativen Besetzungsregelungen * wird die Mindestzahl von Beschäftigten für einen bestimmten Tätigkeitsbereich festgelegt. Klassisches Beispiel sind die Anhänge des MTV für die gewerblichen Arbeitnehmer der Druckindustrie i. d. F. vom 2. 8. 1974. Diese Vorschriften legen für bestimmte Berufssparten den produktionstechnischen Arbeitskräftebedarf fest.

Diese von der ursprünglichen Zielrichtung eher auf eine Absicherung berufsbezogener Standards (Einsatz nur entsprechend der beruflichen Qualifikation) ausgerichteten Regelungen von Besetzungsvorschriften haben im Verlauf der gravierenden technologischen Veränderungen in der Druckindustrie eine neue Qualität bekommen. Unter den Bedingungen fortschreitender technischer Umrüstung wirken diese Besetzungsregelungen als Schutz vor Arbeitslosigkeit.

3. MTV für die gewerblichen Arbeitnebmer in der Druckindustrie vom 2. 8. 1974 -Anbänge

Quantitative Mindestbesetzungsvorschriften:

... An Buchdruckrotationsmaschinen sind zu beschäftigen:

an I Druckeinheit I Drucker,

an 2 Druckeinheiten 2 Drucker,

an 3 Druckeinheiten 3 Drucker.

Die Besetzung weiterer Druckeinheiten wird durch Betriebsvereinbarung geregelt.

... Für die Bedienung von Tiefdruckrotationsmaschinen während des Fortdrucks gilt folgende Richtlinie:

An jeder normal ausgestatteten Tiefdruckrotationsmaschine mit s Druckwerken sind zu beschäfrigen bei einer Arbeitsbreite

bis zu $110 \mathrm{~cm}$

von II I $\mathrm{cm}-175 \mathrm{~cm}$

von $176 \mathrm{~cm}-210 \mathrm{~cm}$

2 Drucker,

3 Drucker,

4 Drucker.

Bei einer Arbeitsbreite über $210 \mathrm{~cm}$ ist die Besetzung durch Betriebsvereinbarung zu regeln. In dieser Betriebsvereinbarung dürfen die Besetzungsnormen für die obengenannten Breiten nicht unterschritten werden...

(Anhang Druck)

4 Vgl. hierzu auch den Entwurf der IG Druck und Papier, in: druck + papier 1977, S. Is ff. Das Ziel der IG Druck und Papier, die unbefristete Arbeitsplatzsicherung, konnte nicht durchgesetzt werden. 
c) Ein weiteres Instrument des mittelbaren Kündigungsschutzes sind die tarifvertraglich vereinbarten Mindestarbeitsinhalte. Darin werden für einzelne Arbeitsplätze bestimmte Tätigkeiten vorgeschrieben, um Dequalifizierungsprozessen und arbeitsorganisatorischen Rationalisierungsmaßnahmen sowie damit verbundener Arbeitsplatzunsicherheit entgegenzuwirken.

4. TV über die Einfübrung und Anwendung rechnergesteuerter Textsysteme in der Druckindustrie vom 20. 3. 1978 :

$\$ 15$ Arbeit in der Redaktion

(1) Die Einführung rechnergesteuerter Textsysteme sowie deren Programmierung darf die journalistische Arbeit, insbesondere die inhaltliche und grafische Gestaltungsmöglichkeit der Redaktion nicht beeinträchtigen. Dieser Grundsatz muß in allen Stadien der Planung, Einführung und Anwendung des Systems befolgt werden. Die Wahrung des Redaktionsgeheimnisses muß sichergestellt werden.

(2) Die Arbeit mit Bildschirmgeräten darf von Redakteuren nur zum Lesen und Redigieren verlangt werden. Die Eingabe eigener Texte kann von ihnen nur insoweit verlangt werden, als es sich um deren erstmalige Niederschrift handelt und eine entsprechende Tätigkeit vor Einführung des rechnergesteuerten Textsystems redaktionsüblich war. Die Eingabe fremder Texte darf von Redakteuren nicht verlangt werden. Die Arbeitsplatzsicherung nach $\$ 2$ darf durch die vorstehende Regelung nicht eingeschränkt werden.

(3) Bei Einführung eines rechnergesteuerten Textsystems kann der einzelne Redakteur die Arbeit mit dem Bildschirmgerät ablehnen, wenn diese Arbeit für ihn unzumutbar wäre. Die Geltendmachung der Unzumutbarkeit und die Stellungnahme des Verlags bedürfen der Schriftform.

(4) Bildschirmgeräte werden nicht als Hilfsmittel zur individuellen Leistungsbeurteilung von Redakteuren eingesetzt.

\section{Weiterbeschäftigung auf einem gleichwertigen bzw. zumutbaren Arbeitsplatz}

In einigen Tarifverträgen (insbesondere bei den Abkommen im öffentlichen Dienst) ist vorgesehen, daß bei Rationalisierungsmaßnahmen im Betrieb bzw. in der Behörde vorhandene Weiterbeschäftigungsmöglichkeiten auf gleichwertigen bzw. zumutbaren Arbeitsplätzen ausgeschöpft werden müssen, bevor eine Entlassung ausgesprochen werden darf. Damit werden rationalisierungsbedingte Kündigungen real erschwert, indem die im Klagefall relevanten kündigungsrechtlichen Entlassungsvoraussetzungen präzisiert und ausgeweitet werden.

Diese tarifvertraglichen Regelungen knüpfen inhaltlich an die von der Rechtsprechung zur Auslegung des $\$$ I Abs. 2 Kündigungsschutzgesetz und die Vorschriften in $\$ I02 Abs. 3 Ziff. 3 BetrVG und $\$ 79$ Abs. I Nr. 3 PersVG (Bund) entwickelten Grundsätze an.

5. TV über den Rationalisierungsschutz für Arbeiter vom 6. 5. 1970 i. d. F. des TV vom 7. II. 1974. Geltungsbereich: MTV für Arbeiter der Gemeinden - BMT - G -.

$\S_{4}$ Arbeitsplatzsicherung

(I) Der Arbeitgeber ist verpflichtet, dem von einer Rationalisierungsmaßnahme betroffenen Arbeiter - ggf. nach Umschulung - einen mindestens gleichwertigen Arbeitsplatz zu sichern. Kann dem Arbeiter kein Arbeitsplatz im Sinne des Unterabsatzes I zur Verfügung gestellt werden, ist der Arbeitgeber verpflichtet, dem Arbeiter - ggf. nach Umschulung - einen anderen zumutbaren Arbeitsplatz anzubieten.

Kann dem Arbeiter kein Arbeitsplatz im Sinne der Unterabsätze I und 2 zur Verfügung gestellt werden, ist der Arbeitgeber verpflichtet, sich um einen zumutbaren Arbeitsplatz bei einem anderen unter den BMT-G fallenden Arbeitgeber an demselben Ort zu bemühen.

Kann dem Arbeiter kein Arbeitsplatz im Sinne der Unterabsätze $\mathrm{x}$ bis 3 zur Verfügung gestellt werden, ist der Arbeitgeber verpflichtet, sich um einen zumutbaren Arbeitsplatz bei einem anderen Arbeitgeber des öffentlichen Dienstes an demselben Ort zu bemühen.

(2) Der Arbeiter ist verpflichtet, einen ihm vom Arbeitgeber angebotenen Arbeitsplatz im Sinne des Absatzes I anzunehmen. 
(3) Kündigungen mit dem Ziel der Beendigung des Arbeitsverhältnisses dürfen bei Arbeitern, die beim Wechsel der Beschäftigung eine Beschäftigungszeit von weniger als is Jahren zurückgelegt haben, nur dann ausgesprochen werden, wenn für den Arbeiter kein Arbeitsplatz nach Absatz I zur Verfügung steht oder der Arbeiter einen ihm angebotenen Arbeitsplatz im Sinne des Absatzes I nicht annimmt. Die Kündigungsfrist beträgt in diesem Fall drei Monate zum Vierteljahresschluß.

Bei Arbeitern, die beim Wechsel der Beschäftigung eine Beschäftigungszeit von mehr als is Jahren zurückgelegt haben, dürfen Kündigungen mit dem Ziel der Beendigung des Arbeitsverhältnisses im Zusammenhang mit Rationalisierungsmaßnahmen nur dann ausgesprochen werden, wenn der Arbeiter einen gleichwertigen Arbeitsplatz bei demselben oder bei einem anderen unter den BMT-G fallenden Arbeitgeber ablehnt oder wenn ihm aus in seiner Person liegenden Gründen ein solcher Arbeitsplatz nicht angeboten werden kann. Für diese Kündigung aus wichtigem Grund gilt Unterabsatz I Satz 2 entsprechend.

6. TV Nr. 306 (Arbeiter) und TV Nr. 307 (Angestellte) über den Rationalisierungsschutz der Arbeiter und Angestellten bei der Bundespost vom 2. 5. bzw. 4. 5. 1972.

\$s Arbeitsplatzsicherung

(I) Der Arbeiter ist verpflichtet, einen ihm angebotenen zumutbaren anderen Arbeitsplatz - ggf. auch an einem anderen Beschäftigungsort - anzunehmen und sich ggf. einer für ihn zumutbaren Umschulungsmaßnahme zu unterziehen.

Die Prüfung der Zumutbarkeit erfolgt im Einvernehmen mit der zuständigen Personalvertretung. Dabei sind u. a. nachstehende Gesichtspunkte zu berücksichtigen:

Alter, Familienstand, Zahl der Kinder, sonstige unterhaltsberechtigte Personen im Haushalt des Arbeiters, Schwerbeschädigung des Arbeiters, Eigenheim, Eigentumswohnung, Umschulung der Kinder, Verlust von Ausbildungsmöglichkeiten der Kinder, Verlust von Beschäftigungsstellen der Familienmitglieder, Betreuung von Eltern oder Großeltern, zusätzliche Fahrtdauer zur neuen Arbeitsstelle, zusätzliche Fahrkosten.

Bei Vorliegen gleicher oben angeführter Gesichtspunkte ist grundsätzlich dem lebensjüngeren Arbeiter gegenüber dem lebensälteren Beschäftigten die Umsetzung zuzumuten.

Eine Umschulung ist für den Arbeiter in der Regel dann unzumutbar, wenn er das so. Lebensjahr vollendet hat.

Lehnt der Arbeiter ein zumutbares Angebot ab, so verliert er die Ansprüche aus diesem Tarifvertrag.

(2) Die Deutsche Bundespost ist verpflichtet, dem von einer Rationalisierungsmaßnahme betroffenen Arbeiter einen anderen gleichwertigen und zumutbaren Arbeitsplatz - ggf. auch an einem anderen Beschäftigungsort - anzubieten. Ist dies ausnahmsweise im Einzelfall nicht sogleich möglich, so ist die Deutsche Bundespost verpflichtet, dem Arbeiter einen anderen zumutbaren Arbeitsplatz mit geringerer Entlohnung - ggf. auch an einem anderen Beschäftigungsort - anzubieten.

(3) Zur Gewährleistung der Grundsätze der Präambel werden bei den OPDn und dem BPM Personalausgleichsstellen eingerichtet.

(4) Soweit für eine Umsetzung nach Abs. 2 Satz I fachliche Voraussetzungen fehlen, die durch Umschulungen geschaffen werden können, veranlaßt die Deutsche Bundespost innerhalb der Lohnsicherungsfristen oder ggf. unter Verlängerung dieser Fristen die Umschulung zum Erwerb der Qualifikation für die neue Tätigkeit.

\section{Schutz älterer Arbeitnehmer}

Eine weitere Gruppe von Arbeitsplatzsicherungsregelungen stellt auf die älteren Beschäftigten ab. Die entsprechenden Schutzregelungen sind an das Lebensalter sowie die Dauer der Betriebszugehörigkeit gebunden. Ziel dieser Vereinbarungen ist es, diejenigen stärker zu schützen, die ein besonderes Arbeitsmarktrisiko tragen. Durch die Zuordnung des Schutzes auf eine bestimmte Gruppe der Beschäftigten ist zunächst nur eine Verteilung der Arbeitsplatzrisiken von einer auf andere Gruppen vorgenommen worden. Dabei verbleibt diese Differenzierung zwischen den Gruppen (ältere Beschäftigte/andere Beschäftigte) auf der Ebene der in $\$$ r Abs. 3 S. I des

s L.AG Düsseldorf in DB 52,$740 ;$ ArbG Ulm in AP I 5 zu $\$$ I KSchG. 
Kündigungsschutzgesetzes vorgesehenen Regelung der sozialen Auswahl, wobei die Rechtsprechung zu den Kriterien der sozialen Auswahl vor allem auf Lebensalter', Betriebszugehörigkeit ${ }^{6}$, Berufsaussichten auf dem Arbeitsmarkt ${ }^{7}$ abstellt. Daneben spielen die sog. "sozialen « Aspekte (Familienstand, Gesundheitsschädigungen, bestehende Unterhaltspflichten) eine Rolle.

Die sehr starke Zuordnung der Schutzregelungen auf ältere Beschäftigte kann dazu führen, die Einstellung dieser Beschäftigungsgruppe zu vermeiden. Die intendierte Schutzabsicht würde dann genau das Gegenteil bewirken: statt Verringerung eine Vergrößerung des Arbeitsmarktrisikos.

\section{MTV für die Arbeitnebmer der Zigarettenindustrie vom 18. 12. 1975.}

$\$ 14$ Schutz älterer Arbeitnehmer

Arbeitnehmer, die das 50 . Lebensjahr vollendet haben, dem Unternehmen mindestens 10 Jahre angehören und bei denen die Summe von Lebensalters- und Betriebszugehörigkeitsjahren mindestens 6s ergibt, genießen erhöhten Schutz:

I. Eine ordentliche Kündigung aus betriebsbedingten Gründen ist ausgeschlossen.

Das gilt nicht für Kündigungen

a) im Rahmen einer Massenentlassung gemäß $\$ 17 \mathrm{KSchG}$ oder eines Sozialplanes gemäß $\$$ I I 2 BetrVG;

b) in Unternehmen mit weniger als roo Arbeitnehmern wegen nachhaltiger Absatzminderung. 2. Eine Änderungskündigung durch den Arbeitgeber aus betriebsbedingten Gründen zur Versetzung auf einen niedriger bewerteten Arbeitsplatz ist zulässig; in diesem Fall und bei einer solchen Änderungskündigung wegen gesundheitlich bedingter Leistungsminderung ist der Arbeitnehmer während der gesamten Dauer des Arbeitsverhältnisses - aber längstens bis zu dem Zeitpunkt, zu dem eine Rente aus der gesetzlichen Rentenversicherung gewährt werden kann - nach der am Tage vor der Versetzung maßgeblichen Tarifgruppe zu bezahlen. 3. Maßgebend für die Berechnung des Lebensalters und der Betriebszugehörigkeit ist der Tag des Anhörungsantrages an den Betriebsrat.

\section{Verlängerung der Kündigungsfristen}

Die Verlängerung der Kündigungsfristen zielt auf eine zeitlich bemessene Hinausschiebung von Entlassungen. Regelungen sind hierzu in verschiedenen Tarifverträgen und Vereinbarungen vorgesehen, vor allem in den Manteltarifverträgen der einzelnen Bereiche. Für den betroffenen Beschäftigten soll sichergestellt werden, daß eine "angemessene Úbergangszeit « bis zur Aufnahme einer neuen Tätigkeit besteht.

Diese Intention kann allerdings dann ins Leere laufen, wenn die strukturelle bzw. regionale Arbeitsmarktsituation keine Möglichkeit des Arbeitsplatzwechsels zuläßt. Eine Verlängerung der Kündigungsfristen wird dann lediglich zur zeitlichen Verzögerung der Arbeitslosigkeit und den damit verbundenen ökonomischen und sozialen Auswirkungen.

8. Rationalisierungsschutztarifvertrag für die gewerblichen Arbeitnehmer der Textilindustrie vam 30. 4. 1969.

\$6. I. Für Änderungskündigungen, die bei Umsetzungen von Arbeitnehmern unter Minderung des Arbeitsverdienstes infolge von Rationalisierungsmaßnahmen notwendig werden, gelten die folgenden Kündigungsfristen:

6 Herschel in Anmerk. zu EzA Nr. 3 zu $§$ I KSchG »Betriebsbedingte Kündigung*.

7 LAG Düsseldorf in DB $\{2,828$. 


\begin{tabular}{lcccc} 
Lebensalter & ab 10 & ab is & $\begin{array}{c}\text { Betriebszugehörigkeit } \\
\text { ab 20 }\end{array}$ & ab 25 Jahre \\
\hline ab 40 Jahre & 8 & I & 16 & 20 Wochen \\
ab 45 Jahre & 10 & 14 & 18 & 22 Wochen \\
ab 50 Jahre & 14 & 18 & 22 & 26 Wochen \\
ab 55 Jahre & 18 & 22 & 26 & 32 Wochen \\
ab 60 Jahre & 22 & 26 & 32 & 39 Wochen
\end{tabular}

Maßgebend sind das Lebensalter und die Betriebszugehörigkeit des Arbeitnehmers bei Ausspruch der Änderungskündigung.

\$7. I. Bei Entlassungen, die infolge von Rationalisierungsmaßnahmen notwendig werden, gelten die Kündigungsfristen der Tabelle in $\$ 6$, Ziff. I.

\section{Umschulung}

Umschulungsmaßnahmen sollen »freigesetzten « Beschäftigten durch Weiterbildung die auf anderen Arbeitsplätzen geforderten Qualifikationen vermitteln. Sie sollen dadurch zur Arbeitsplatzsicherung beitragen. Für die Beurteilung der entsprechenden Regelungen sind folgende Gesichtspunkte von Bedeutung:

- Der Kreis der Beschäftigten, die eine Umschulung in Anspruch nehmen können, ist durch die jeweiligen Voraussetzungen (z. B. Verlust des Arbeitsplatzes, "nicht an neuen Geräten eingesetzt werden können ", "nach Maßgabe ihrer persönlichen und fachlichen Eignung «, "nach Maßgabe der neu zu besetzenden Arbeitsplätze«) stark eingeschränkt. Diese Vielzahl von Voraussetzungen macht die Umschulung zu einem Instrument, das allein seitens der Unternehmen je nach Bedarf eingesetzt werden $\mathrm{kann}^{8}$ : Anpassungsfortbildung zur Flexibilisierung des jeweils benötigten Arbeitskräftepotentials.

- Durch die zeitliche Befristung ( 3 bis maximal 12 Monate) ist kaum eine umfassende Qualifizierung für neue Tätigkeitsbereiche oder gar neue Berufsqualifizierung möglich. Die zeitliche Befristung verweist auf kurzfristige Anlernprozesse. Aspekte der langfristigen Entwicklung des Arbeitsprozesses und dementsprechende Qualifikationsanforderungen werden kurzfristigen Verwertungsnotwendigkeiten untergeordnet.

- Die Kostenregelungen sind sehr unterschiedlich. Teilweise sollen alle anfallenden Kosten (Weiterzahlung des Lohns, Umschulungskosten) vom Arbeitgeber gezahlt werden, teilweise nur die Differenzbeträge zwischen entstehenden Gesamtkosten und in Anspruch zu nehmenden Leistungen.

- Durch Bestimmungen wie »Unzulässigkeit der willkürlichen Verweigerung der Zustimmung zu zumutbaren Umschulungsmaßnahmen « und "Anrechnung des fortgezahlten Arbeitsentgelts bei schuldhafter Erfolglosigkeit der Umschulung « oder sogar Kostenrückzahlungsklauseln wird der Mobilitätsdruck auf die Beschäftigten erheblich erhöht.

\footnotetext{
8 Geringe Einflußmöglichkeiten bestehen im Rahmen des Beteiligungsverfahrens bei Kündigungen für Personalvertretung ( $\$ 79$ Abs. I Nr. 3 Bundespersonalvertretungsgesetz) und Betriebsrat $(\$ 102$ Abs. 3 Nr. ${ }_{4}$ BetriebsVG).

Durch die Heranziehung des Einspruchstatbestands "Weiterbeschäftigung des Arbeitnehmers nach zumutbaren Umschulungs- und Fortbildungsmöglichkeiten « bestehen Interventionsmöglichkeiten. Wie Erd, Verrechtlichung industrieller Konflikte, S. 76, festgestellt hat, verlangt diese Interventionsmöglichkeit umfassende Kenntnisse über langfristige Entwicklungen der Arbeitsplätze und Besetzungsmöglichkeiten, um jeweils bezogen auf den Einzelfall den Arbeitsplatz zu sichern.
} 
9. TV ̈̈ber Rationalisierungsschutz für Arbeiter des Bundes und der Länder vom 29. 10. 1971 i.d.F. des Anderungs-TV Nr. 2 vom 7. I. 1974. Geltungsbereich: MTV für Arbeiter des Bundes - MTB - und MTV für die Arbeiter der Länder - MTL II -

\s Umschulungsmaßnahmen

(r) Zur Erfüllung der sich aus $\S_{4}$ Abs. I Unterabs. I und 2 ergebenden Verpflichtungen soll der Arbeitgeber, soweit erforderlich, Umschulungsmaßnahmen veranlassen oder auf seine Kosten durchführen. Der Arbeiter darf seine Zustimmung zu einer zumutbaren Umschulungsmaßnahme nicht willkürlich verweigern.

(2) Der Arbeiter ist für die zur Umschulung erforderliche Zeit von der Arbeit unter Zahlung des Urlaubslohnes $(\$ 48$ Abs. 2 bis 6 MTB H/MTL II) freizustellen. Wird durch die Umschulung die durchschnittliche regelmäßige wöchentliche Arbeitszeit überschritten, ist ihm ein entsprechender Freizeitausgleich bis zur Dauer der vereinbarten regelmäßigen wöchentlichen Arbeitszeit zu gewähren. Der Urlaubslohn wird längstens für die Dauer von sechs Monaten gezahlt. Eine Verlängerung um längstens weitere sechs Monate ist möglich.

(3) Setzt der Arbeiter nach der Umschulung aus einem von ihm zu vertretenden Grunde das Arbeitsverhältnis nicht für einen mindestens der Dauer der Umschulung entsprechenden Zeitraum fort, ist der Arbeitgeber berechtigt, den nach Absatz 2 gezahlten Urlaubslohn und die Kosten der Umschulung zurückzufordern.

10. TV über die Einfübrung und Anwendung rechnergesteuerter Textsysteme in der Druckindustrie vom 20.3. 1978

$\$ 7$ Betriebliche Umschulung

(I) Arbeitnehmer, die durch die Einführung des rechnergesteuerten Textsystems ihren Arbeitsplatz verlieren und nicht an den neuen Geräten eingesetzt oder in anderer Weise beschäftigt werden können, werden nach Maßgabe ihrer fachlichen und persönlichen Eignung sowie entsprechend der Zahl neu zu besetzender Arbeitsplätze im Betrieb bzw. Unternehmen umgeschult. Die Umschulungsmaßnahmen müssen dem Betrieb bzw. Unternehmen und dem Arbeitnehmer, auch was ihre Dauer angeht, zumutbar sein. Umschulungen innerhalb des technischen Bereiches sind auf 13 Wochen begrenzt.

(2) Für die Dauer der innerbetrieblichen Umschulung wird das vereinbarte Arbeitsentgelt, längstens für einen Zeitraum von 13 Wochen bzw. 3 Monaten, weitergezahlt. Dauert die innerbetriebliche Umschulung länger als 3 Monate, so ist die Frage des Entgelts für den über 3 Monate hinausgehenden Zeitraum einzelvertraglich zu vereinbaren. Zuschläge für während der Umschulung tatsächlich geleistete Nacht-, Sonn- und Feiertagsarbeit sind zu zahlen.

(3) Führt die innerbetriebliche Umschulung durch schuldhaftes Verhalten des Arbeitnehmers nicht zum Erfolg, so wird das gemäß Abs. 2 fortgezahlte Arbeitsentgelt auf die Abfindung gemäß Sozialplan bzw. $\ 10$ dieses Tarifvertrages angerechnet.

(4) Uber die erfolgreich beendete Umschulung erhält der Arbeitnehmer eine Bescheinigung, aus der Art und Umfang der Umschulung hervorgehen müssen.

\section{Kündigungsschutz wäbrend Einarbeitungszeit}

Regelungen dieser Art sollen während der Umschulungs- und der sich daran anschließenden Einarbeitungszeit Kündigungen wegen auftretender Schlecht- oder unzureichenden Leistungen ausschließen. Sie lehnen sich dabei an die von der Rechtsprechung entwickelten Kriterien zur Kündigung aus »im Verhalten des Arbeitnehmers « liegenden Gründen an. In jedem Falle ist eine vorherige Abmahnung ${ }^{9}$ des Beschäftigten erforderlich, so daß zeitlich gesehen eine Kündigung erst nach einer bestimmten Frist ausgesprochen werden darf. Auch im Hinblick auf die vom Gericht im Prozeßfall vorzunehmende Interessenabwägung wird eine Kündigung während der Einarbeitungszeit kaum durchgesetzt werden. 


\section{$\$ 6$ Besonderer Kündigungsschutz}

Wird dem Arbeitnehmer als Folge einer Rationalisierungsmaßnahme eine neue Tätigkeit übertragen, darf das Arbeitsverhältnis während der ersten 6 Monate dieser Tätigkeit vom Arbeitgeber weder aus betriebsbedingten Gründen noch wegen mangelnder Eignung gekündigt werden.

\section{Wiedereinstellungspriorität}

Diese Form der Sicherung soll entlassenen Beschäftigten die Möglichkeit eröffnen, bei ihrem bisherigen Arbeitgeber bei freigewordenen Arbeitsplätzen bevorzugt eingestellt zu werden. Abgesehen von den damit verbundenen Informationsproblemen ist fraglich, ob durch diese Regelung ein besonderer Konkurrenzvorteil auf dem Arbeitsmarkt für von Rationalisierungsmaßnahmen betroffene Arbeitnehmer entsteht. Beispiele aus Bereichen der Wirtschaft und des privaten Dienstleistungsbereiches (Tiefbau, private Naßbaggerei) geben der Vermutung Nahrung, daß es sich hierbei nicht nur um die Schaffung eines beschäftigungspolitischen Instrumentariums zur Sicherung von Beschäftigungsmöglichkeiten von Gruppen auf dem Arbeitsmarkt, sondern u. U. bloß um die zeitweilige Abwälzung von Kosten auf die Solidargemeinschaft (Sozial- und Arbeitslosenversicherung) geht. Betrachtet man den Regelungszweck dieser Vorschriften unter diesem Aspekt, so sollen qualifizierte, aber momentan (aus welchen Gründen auch immer) nicht gebrauchte Arbeitnehmer »motiviert« werden, zum alten Arbeitgeber zurückzukehren.

12. TV ̈̈ber den Rationalisierungsschutz für Arbeiter vom 6. 5. 1970 i. d. F. des TV vom 7. II. 1974. Geltungsbereich: MTV für Arbeiter der Gemeinden - BMT-G

$\$ 4$ (4) Der Arbeiter, der auf Veranlassung des Arbeitgebers im gegenseitigen Einvernehmen oder aufgrund einer Kündigung durch den Arbeitgeber aus dem Arbeitsverhältnis ausgeschieden ist, soll auf Antrag bevorzugt wieder eingestellt werden, wenn ein für ihn geeigneter Arbeitsplatz zur Verfügung steht.

\section{Zablung von Abfindungen}

In vielen Rationalisierungsschutzabkommen ist die Zahlung von Abfindungen als Ersatz für den Verlust eines Arbeitsplatzes vorgesehen. Die Höhe der Abfindungszahlung bemißt sich in der Regel nach der Dauer der Betriebszugehörigkeit.

$\mathrm{Da}$ die Abfindungen zum Teil auch bei einvernehmlichem Ausscheiden bezahlt werden, sind sie auch ein möglicher Köder für die Beschäftigten, selbst zu kündigen.

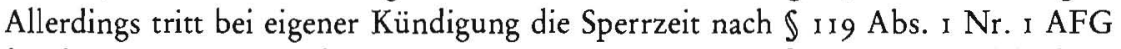
für die Zahlung von Arbeitslosenunterstützung ein, so daß ein Teil der Abfindung auf diese Weise wieder aufgezehrt wird (vgl. auch $\$$ II 7 AFG).

Die Möglichkeit der Abfindungszahlung war zu Beginn der tarifvertraglichen Folgenbewältigung von Rationalisierungsmaßnahmen durch finanzielle Kompensation des rationalisierungsbedingten Personalabbaus ohne Widerstände durchzusetzen. Ähnlich wie beim gerichtlichen Kündigungsschutzverfahren, wo das Ziel des Bestandsschutzes der Arbeitsverhältnisse durch die Inanspruchnahme von Abfindungszahlungen nach den $\$ \$ 9$, Io KSchG verdrängt worden war ${ }^{10}$, waren die damit verbundenen sozialen und ökonomischen Folgen nicht so einschneidend, da die betroffenen Beschäftigten meist bereits einen neuen Arbeitsplatz in Aussicht hatten oder sich kurzfristig anderweitige Beschäftigungsmöglichkeiten erhofften.

10 Klees, B., Wie rechtsstaatlich ist die Arbeitsgerichtsbarkeit heute?, in: DuR 1975, S. 26 ff. 
Bei zunehmender struktureller und regionaler Arbeitslosigkeit greift diese Kompensationsstrategie immer weniger. Die Inanspruchnahme einer Abfindung mit dem Ziel, mit dieser "Übergangshilfe« einen neuen Arbeitsplatz zu suchen, erweist sich als unkalkulierbares Risiko für die betroffenen Beschäftigten.

13. TV über Einfübrung und Anwendung rechnergesteuerter Textsysteme in der Druckindustrie vom 20. 3. 1978

$\S 8$ Mobilitätshilfe

Arbeitnehmer, insbesondere Schriftsetzer, deren Arbeitsplätze durch Einführung des rechnergesteuerten Textsystems entfallen und die bereit sind, einen ihnen nachgewiesenen Arbeitsplatz in ihrem Beruf an einem anderen Ort in der Bundesrepublik Deutschland einschließlich Berlin (West) anzunehmen, erhalten vom abgebenden Unternehmen folgende finanzielle Brutto-Unterstützung (Mobilitätshilfe):

a) eine Pauschale in Höhe von $150 \%$ der erforderlichen Umzugskosten (Nachweis durch Rechnung der Spedition) und

b) die nachgewiesene Differenz vom alten vereinbarten Stundenlohn/Gehalt und dem am neuen Arbeitsplatz gezahlten Stundenlohn-Gehalt für I Jahr auf der Basis von 173 Stunden monatlich.

14. TV über den Rationalisierungsschutz für Beschäftigte in der Energieversorgung BadenWürtemberg vom 22. 6. 1977

$\$ 9$ Beendigung des Arbeitsverhältnisses - Abfindung

(9.I) Wenn eine Weiterbeschäftigung im Unternehmen nicht mehr möglich ist und auch die Voraussetzungen des $₫ 8$ nicht vorliegen, kann das Arbeitsverhältnis gekündigt werden.

(9.2) Für den Verlust des Arbeitsplatzes erhält der Arbeitnehmer eine Abfindung nach folgender Staffel:

Bei einer Betriebszugehörigkeit

von mehr als 3 Jahren 2 Monatsvergütungen

von mehr als 5 Jahren 3 Monatsvergütungen

von mehr als io Jahren 6 Monatsvergütungen

von mehr als is Jahren 9 Monatsvergütungen

von mehr als 20 Jahren 12 Monatsvergütungen

(9.3) Keinen Anspruch auf eine Abfindung hat ein Arbeitnehmer, der die Durchführung der nach diesem Abkommen für seine Weiterbeschäftigung vorgesehenen personellen Einzelmaßnahmen ablehnt oder eine bereits eingeleitete Umschulung ohne berechtigten Grund und ohne Einwilligung des Arbeitgebers vorzeitig abbricht.

\section{Ausdehnung mit Mitspracherechten}

Hierbei wird die Mitwirkung des Betriebsrats bei personellen Angelegenheiten zu einer gleichgewichtigen Beteiligung ausgeweitet.

15. MTV für die Arbeiter der Zigarettenindustrie vom I8. 12. I975

$\$ 16$ Mitbestimmung und Mitwirkung des Betriebsrats in personellen Angelegenheiten und bei Rationalisierungsmaßnahmen

I. Einstellung und Entlassung von Arbeitnehmern erfolgen in Übereinstimmung zwischen Betriebsleitung und Betriebsrat.

$\$$ i 7 Schlichtung von Streitigkeiten

2. In den Fällen des $\ 16$ Nrn. 1, 3 b, 4 e entscheidet eine Schiedsstelle verbindlich. Sie besteht aus zwei Arbeitgeber- und zwei Arbeitnehmervertretern. Der Vorsitz wechselt von Fall zu Fall zwischen den Parteien.

\section{Einkommensicherung}

Einkommenssicherung bezieht sich auf die vorübergehende bzw. ständige Festschreibung der bestehenden Einkommenshöhe: 

rung,

- endgültige Verdienstsicherung für Ältere,

- Nebenleistungssicherung (Zuschläge), Versorgungssicherung,

- Mehraufwandserstattung.

\section{Besitzstandssicherung durch zeitweilige Verdienstabsicherung bei Abgruppierung}

16. TV zur Sicherung der Eingruppierung und zur Verdienstssicherung bei Abgruppierung für Arbeiter und Angestellte in der Metallindustrie Nordwürttemberg/Nordbaden vom 3. 4 . $1978 .^{\text {" }}$

\$4.2. Eine Abgruppierung ist erst zulässig, wenn eine Umsetzung nach $\$ 3.1$ bzw. eine Umschulung nach $\$ 3.2$ nicht möglich ist.

$\$ 4.3$. Ein Arbeiter kann höchstens um zwei Lohn-/Arbeitswertgruppen, ein Angestellter höchstens um eine Gehaltsgruppe abgruppiert werden. In der niedrigeren Gehaltsgruppe ist er in das gleiche Beschäftigungsjahr einzustufen wie in der höheren Gehaltsgruppe. In der Arbeitswertgruppe XII entsprechen 3 Arbeitswerte einer Arbeitswertgruppe im Sinne von Satz I.

$\$$ 5.1. Bei einer Abgruppierung erhält der Arbeitnehmer, sofern er mindestens 6 Monate in der bisherigen oder einer höheren Lohn-/Arbeitswert-/Gehaltsgruppe im Unternehmen eingruppiert war, nach Ablauf der Frist für eine Änderungskündigung und/oder nach Ablauf einer Ankündigungsfrist für die Abgruppierung einen Verdienstausgleich für die Dauer von 18 Monaten, abzüglich des Zeitraumes der Kündigungsfrist und/oder der Ankündigungsfrist.

$\$ 5.3$. Während der Frist gemäß $\$ 5.1$ nimmt der Verdienstausgleich an der Erhöhung des Tariflohnes bzw. Tarifgehaltes teil.

\$5.4. Nach Ablauf der Frist gemäß \$ 5.I kann die erste Erhöhung des Tariflohnes bzw. Tarifgehaltes auf den Verdienstausgleich angerechnet werden. Alle nachfolgenden Erhöhungen des Tariflohnes/Tarifgehaltes können bis zu 50\% auf den Verdienstausgleich angerechnet werden.

\section{Endgültige Verdienstsicherung für ältere Arbeitnehmer}

17. MTV für die gewerblichen Arbeitnebmer der Metallindustrie Nordwürttemberg/-baden vom 20. 10. 1973

\$9.r. Arbeitnehmer, die im 5s. Lebensjahr stehen oder älter sind und dem Betrieb oder Unternehmen wenigstens I Jahr lang angehören, haben Anspruch auf den Verdienst, der aus dem Durchschnittsverdienst der letzten I 2 voll gearbeiteten Kalendermonate errechnet wird. Tariflohnerhöhungen steigern den Verdienst entsprechend.

\section{Nebenleistungssicherung}

Zulagen als Lohnbestandteil, die sich auf bestimmte Belastungen im Arbeitsprozeß beziehen, sind immer gefährdet, wenn durch Rationalisierungsmaßnahmen die konkreten Tätigkeiten verändert werden.

18. TV Nr. 306 (Arbeiter) und TV Nr. 307 (Angestellte) über den Rationalisierungsschutz der Arbeiter und Angestellte bei der Bundespost vom 2. 5. bzw 4. 5. 1972

$\$ 4$ Sicherung der Erschwerniszuschläge

(1) Werden Arbeitern, die die Anspruchsvoraussetzungen des $\$ 2$ erfüllen, infolge einer Rationalisierungsmaßnahme andere Tätigkeiten übertragen, bei der die bisher gezahlten Erschwerniszuschläge gemäß Anlage 4 TV Arb ganz oder teilweise wegfallen, so erhalten sie eine Sicherung, wenn sie in den der Ubertragung der anderen Tätigkeit vorausgegangenen zwei

II Der Entwurf vom I3. 4. 1977 sah vor, daß cine kollektive Absicherung (Sicherung des Lohn- und Gehaltsgruppennjveaus des Betriebes) erreicht werden sollte. Vgl. hierzu: Hildebrandr, E., Der Tarifkampf in der metallverarbeitenden Industrie 1978 , in: Jacobi, O., u. a., Arbeiterinteressen gegen Sozialpartnerschaft, Berlin 1979, S. $60 \mathrm{ff}$. 
Jahre überwiegend und auch noch innerhalb der letzten drei Monate Erschwerniszuschläge erhalten haben.

(2) Hierbei erhält der Arbeiter für jede Lohnstunde sowie für Zeiten mit Lohnanspruch, in denen er keine Arbeit leistet, den Betrag, der sich aus dem vor der Ubertragung der anderen Tätigkeiten maßgeblichen v. H.-Satz des Zeitlohnzuschlages nach $\$ 1_{4}$ Abs. 6 letzter Unterabs. TV Arb, vermindert um 3,3 v. H., ergibt.

(3) Dies gilt nicht für Lohnmonate, in denen der Arbeiter aufgrund der neuen Tätigkeit insgesamt einen höheren Anspruch auf Erschwerniszuschläge nach Anlage 4 TV Arb erwirbt.

(4) Die Sicherung wird für folgende Zeiträume gewährt:

a) Vollbeschäftigten Arbeitern mit einer Postdienstzeit von

2 bis s Jahren für die Dauer von Is Monaten,

5 bis 8 Jahren für die Dauer von 22 Monaten,

mehr als 8 Jahren für die Dauer von 28 Monaten.

b) Nichtvollbeschäftigten Arbeitern mit einer Postdienstzeit von

5 bis 8 Jahren für die Dauer von II Monaten,

mehr als 8 Jahren für die Dauer von 14 Monaten.

c) Unkündbaren vollbeschäftigten Arbeitern wird die Sicherung für die Dauer von 36 Monaten, oder, sofern sie das 60. Lebensjahr vollendet haben, bis zur Beendigung des Arbeitsverhältnisses gewährt.

(s) Die Sicherung wird vom Beginn des Lohnmonats an gewährt, der auf die Übertragung der anderen Tätigkeiten folgt.

Mebraufwandserstattung

Mehraufwandserstattung soll versetzten Beschäftigten eine gewisse Kostenentlastung bei rationalisierungsbedingten Maßnahmen bringen.

Dabei handelt es sich um eine Form der zweckgebundenen Abfindung.

19. TV über den Rationalisierungsschutz für Beschäftigte in der Energieversorgung BadenWürttemberg vom 22. 6. 1977

$\$ 6$ Ortswechselausgleich

(6.1) Erfolgt die Versetzung an einen anderen Arbeitsort und erwächst dem betroffenen Arbeitnehmer dadurch ein finanzieller Mehraufwand, so erhält er folgenden Ortswechselausgleich:

a) Trennungsentschädigung

Mehraufwand für Verpflegung in Höhe von DM I,$-/$ kalendertäglich

Kosten für Unterkunft in Höhe von DM 7,-/kalendertäglich

bis zu seinem Umzug, in der Regel für die Dauer von 6 Monaten; läßt sich innerhalb dieses

Zeitraumes keine Wohnung beschaffen, so erfolgt die Zahlung bis längstens I 2 Monate.

b) Familienheimfahrten für diesen Zeitraum

bei doppeltem Haushalt $\quad 2 \times$ monatlich

ohne doppelten Haushalt I $\times$ monatlich

mindestens in Höhe der Kosten der öffentlichen Verkehrsmittel.

c) Umzugskosten

In Höhe der nachgewiesenen Transportkosten sowie mit dem Umzug verbundenen notwendigen Nebenkosten bis zu DM rooo,-.

(6.2) Anstelle der Trennungsentschädigung kann ein Fahrtkostenersatz vereinbart werden.

\section{Versorgungssicherung}

Die Versorgungssicherung stellt auf eine Sicherung der erworbenen betrieblichen Versorgungsansprüche ab. Diese Form der Sicherung lehnt sich an die Vorschriften des Betriebsrentengesetzes an (vgl. $\$$ I Abs. I).

Die Versorgungssicherung ist eine Sonderform der Abfindung, da bei »freiwilligem« Ausscheiden eine finanzielle Kompensation für den Verlust des Arbeitsplatzes erfolgt. 
$\$ 9$ Unverfallbarkeit betrieblicher Versorgungsansprüche

Wird das Arbeitsverhältnis aus Anlaß einer Rationalisierungsmaßnahme beendet, besteht Anspruch auf Versorgungsbezüge nach der betrieblichen Ruhegeldregelung. Der Anspruch ruht bis zum Eintritt des Versorgungsfalles.

$\$ 10$ Versetzung in den Ruhestand

In beiderseitigem Einvernehmen kann eine vorzeitige Versetzung in den Ruhestand mit Anspruch auf Versorgungsbezüge nach der Ruhegeldregelung vereinbart werden.

Hans-Henning Lübr

\section{Erklärung des Vorstands des Republikanischen Anwaltsvereins*}

Der Republikanische Anwaltsverein begrüßt den Gesetzesvorschlag »Die Verteidigung “ (Karlsruhe, 1979, C. F. Müller Verlag), den die im Arbeitskreis Strafprozeßrechtsreform zusammengeschlossenen Strafrechtsprofessoren Bemmann, Grünwald, Hassemer, Kraus, Lüderssen, Naucke, Rudolphi und Welp vorgelegt haben. Die Vorlage, die "Bedeutung als Modell einer kriminalpolitisch und dogmatisch grundlegenden, in tragfähigen Kompromissen auslaufenden Gesetzesdiskussion « beansprucht, ist eine Herausforderung an Bundesregierung und Parlament, die Einschränkung der Verteidigungsrechte durch die seit Ende 1974 begonnene Serie von Änderungen der Strafprozeßordnung zu überdenken und rückgängig zu machen.

Im Strafprozeß werden die Grundrechte unmittelbar angewandt; denn jede einzelne Zwangsbefugnis der Staatsanwaltschaft und jedes prozessuale Recht des Beschuldigten stellt eine Grenzziehung zwischen Staatsgewalt und dem Recht des Einzelnen dar. Das Strafprozeßrecht ist deshalb richtig vom Bundesverfassungsgericht als angewandtes Verfassungsrecht bezeichnet worden. Die seit Ende 1974 festzustellende Tendenz durchkreuzt nicht nur die 1964 begonnene Reform des Strafverfahrensrechts, sondern hat eindeutig die Gewichte im Strafprozeß zugunsten der Strafverfolgungsbehörden und zu Lasten der Stellung des Beschuldigten verschoben.

Im Mittelpunkt der strafprozessualen Rechte eines Beschuldigten steht sein Recht auf Verteidigung und auf einen vom Staat unabhängigen Verteidiger. Die in den letzten Jahren gegen die Verteidigung in Kraft getretenen Gesetze gründen in der Auffassung, daß der Rechtsanwalt nicht nur der berufene Vertreter der Interessen seines Mandanten sei, sondern zugleich, notfalls gegen ihn, auch Interessen der staatlichen Strafverfolgung zu vertreten habe. Dieser Auffassung liegt, wie die Strafrechtsprofessoren schreiben, ein autoritäres Staatsverständnis zugrunde, das dem Gemeinwohl entgegenstehende Interessen des Einzelnen nicht anerkennt. Mit den Grundsätzen, die sich aus dem Grundgesetz und der Europäischen Menschenrechtskonvention ergeben, stimmen sie nicht überein.

Wenn der Republikanische Anwaltsverein dem Gesetzesvorschlag auch nicht in allen Punkten zustimmen kann und einige Punkte weiterer Erörterung bedürfen, wie beispielsweise der Vorschlag, einen Ersatzverteidiger zu bestellen, so begrüßt

* Geschäftsstelle, 3000 Hannover, Lärchenstr. 2. 